\title{
ANÁLISE DA CONCENTRAÇÃO DE SICAM-1 EM CRIANÇAS E ADOLESCENTES COM PROCESSO INFLAMATÓRIO COM DESVIO NUCLEAR À ESQUERDA
}

\author{
Natalie Cristini Peliser ${ }^{1}$ \\ Paulo Roberto Merisio ${ }^{2}$
}

PELISER, N. C.; MERISIO, P. R. Análise da concentração de SICAM-1 em crianças e adolescentes com processo inflamatório com desvio nuclear à esquerda. Arq. Cienc. Saúde UNIPAR, Umuarama, v. 19, n. 2, p, 89-93, maio/ago. 2015

\begin{abstract}
RESUMO: Os neutrófilos são fagócitos e atuam no processo de defesa do organismo iniciando a cascata inflamatória quando ativados. Este processo envolve vários fatores, entre eles a liberação de bastonetes e o aumento na concentração de sICAM-1. Verificou-se o estado de ativação do endotélio em 37 pacientes de 0 a 18 anos sem doenças crônicas, de modo a investigar um possível aumento de sICAM-1 com o aumento de bastonetes. Os bastonetes foram analisados por meio de verificação de prontuário. A determinação das concentrações séricas de sICAM-1 foi realizada por meio da técnica imunoenzimática (ELISA) direto. Foi possível notar que não há correlação entre o aumento de bastonete e o aumento da molécula sICAM-1 ( $\mathrm{r}=0,28)$. Dividindo-se os pacientes em dois grupos, um que possui sICAM-1 alterado e outro que possui sICAM-1 normal, observou-se mediante os dados que estes possuem diferença notável de bastonetes ( $\mathrm{p}=0,047$ ), onde a média de bastonetes é maior no grupo sICAM-1 alterado. A presença de sICAM-1 aumentada, normalmente, está ligada a casos inflamatórios, mas a mudança na sua expressão pode sofrer influência de outros fatores, tais como o uso de anti-inflamatórios esteroidais e tabagismo. Uma possível explicação para não ter ocorrido o aumento de sICAM-1 nos pacientes estudados, é de que estes estejam utilizando medicamentos do tipo anti-inflamatórios esteroidais. Segundo a literatura, a sICAM-1 parece ser um bom marcador para lesão endotelial em pacientes com doenças cardiovasculares, sugere-se então um estudo mais aprofundado para estes casos.
\end{abstract}

PALAVRAS-CHAVES: Neutrófilos. Endotélio. ELISA.

\section{ANALYSIS OF SICAM-1 CONCENTRATION IN CHILDREN AND TEENS WITH INFLAMMATORY PROCESS WITH NUCLEAR SHIFT TO THE LEFT}

\begin{abstract}
S: Neutrophils are phagocytes and act in the body's defense process initiating the inflammatory cascade when activated. This process involves several factors, including the release of band cells and the increase in the concentration of sICAM-1. The activation state of the endothelium in 37 patients from 0 to 18 years old with no chronic diseases was checked, in order to investigate a possible increase of sICAM-1 with increased band cells. The band cells were analyzed through an investigation of medical records. Determination of sICAM-1 serum concentrations was performed by direct enzyme immunoassay (ELISA). No correlation between the increase in band cells and increase the sICAM-1 molecule $(r=0.28)$ could be observed. By dividing the patients into two groups, one with changed sICAM-1 and the other presenting standard sICAM-1, it could be observed by the data they have notable difference in band cells $(p=0.047)$, where the average number of band cells is greater in the group with altered sICAM-1. The presence of increased sICAM-1 is typically linked to inflammatory cases, but the change in their expression can be influenced by other factors, such as the use of steroidal anti-inflammatory medicine and smoking. One possible explanation for the sICAM-1 increase not having occurred in the patients studied is the fact that they are using steroidal anti-inflammatory drugs. According to the literature, sICAM-1 seems to be a good marker for endothelial injury in patients with cardiovascular disease. Therefore, further studies are suggested for these cases.
\end{abstract}

KEYWORDS: Neutrophils; Endothelium; ELISA.

Introdução

Tipicamente, a criança saudável apresenta crescimento e desenvolvimento normais e encontra-se bem entre as ocorrências infecciosas. Na maioria das vezes, as infecções não são prolongadas ou complicadas e ocorrem devido aumento da exposição a agentes infecciosos nos primeiros anos de vida, especialmente durante o outono e o inverno e também pela imaturidade do sistema imunológico (FILIZZOLA et al. 1998; FERREIRA, GROSSEMAN, VIEIRA, 2003). A Maturidade desse sistema se completa na adolescência (RIZZON, 2011).

As células do sistema imune que atuam na defesa do organismo diante dos mais variados patógenos são os leucócitos. Estes estão presentes no sangue em quantidades basais bem definidas, circulam por tempo predeterminado, e vão para os tecidos nos quais desempenham funções específicas (OLIVEIRA, 2007). São divididos em dois grupos: os granulócitos e os agranulócitos (JUNQUEIRA; CARNEIRO,
2008).

Os granulócitos mostram no citoplasma grânulos específicos e tem núcleo de forma irregular. De acordo com a granulação específica, têm-se três tipos de granulócitos: neutrófilos, eosinófilos e basófilos. Os agranulócitos não possuem granulações específicas e tem núcleo com forma mais regular. Há dois tipos de agranulócitos: os linfócitos e os monócitos (JUNQUEIRA; CARNEIRO, 2008). Cada tipo de leucócito possui mecanismo regulatório e de maturação diferentes. Seus níveis de produção são dependentes da necessidade orgânica mais notável (OLIVEIRA, 2007).

Os neutrófilos compõem a maior população de leucócitos presentes na circulação e são mediadores das fases iniciais das respostas inflamatórias (ABBAS, LICHTMAN, POBER, 2002), isto é, atuam na primeira linha de defesa do organismo, fagocitando, matando e digerindo bactérias e fungos. Seus núcleos são formados por dois a cinco lóbulos ligados entre si por finas pontes de cromatina. A célula jovem tem núcleo não segmentado, sendo chamada de neutrófilo 
com núcleo em bastonete ou, simplesmente, bastonete. $\mathrm{Na}$ circulação existe apenas um pequeno número de bastonetes (JUNQUEIRA; CARNEIRO, 2008).

De acordo com as condições normais, os fatores de crescimento estimuladores de colônia de granulócitos e de macrófagos-granulócitos (G-CSF e GM-CSF) estimulam o desenvolvimento de progenitores mielóides, eritóides e megacariócitos. A liberação dos neutrófilos para a circulação sanguínea ocorre após a maturação completa na medula óssea. Já em um processo inflamatório, o GM-CSF regula a quantidade de neutrófilos, aumentando o desenvolvimento de novos progenitores mielóides. Com o aumento de progenitores ocorre uma ampliação na produção e liberação de neutrófilos, essa liberação acelerada pode resultar no aparecimento de neutrófilos bastonetes na circulação (BOTHA et al., 1995; HILL et al., 1995; KUIJPERS; ROOS, 2001).

Contudo, a presença de leucocitose e neutrofilia, com aumento de neutrófilos bastonetes que caracterizam desvio nuclear à esquerda (DNE), é importante para o diagnóstico de quadros infecciosos juntamente com outros fatores, tais como alterações morfológicas de leucócitos (PARAHYBA, SANTANA, ALENCAR, 2000).

Após um estímulo desencadeante inflamatório, as células ativadas do sistema fagocítico mononuclear (monócitos e macrófagos) iniciam a cascata de eventos da resposta inflamatória aguda, secretando citocinas da família da interleucina-1 (IL-1) e fator de necrose tumoral (TNF). Essas moléculas agem especialmente sobre fibroblastos e células endoteliais e causam a liberação de outras que incluem, além das IL-1 e TNF, também IL-6 e IL-8 e as proteínas inflamatória e quimiotática de macrófago. Esta última proteína, em conjunto com a IL-1 e IL-8, atrai para o local da inflamação monócitos e neutrófilos, os quais secretam citocinas que retroalimentam a cascata inflamatória (MORTENSEN, 2001; FIOTTI et al., 1999).

Deste modo, no processo inflamatório os neutrófilos rolam na superfície do endotélio, mediados pela ação das selectinas (E, P e L), depois são ativados mediante a ação de quimiocinas, que fazem que a avidez dos leucócitos pelas integrinas aumente, e aderindo fortemente ao endotélio pormeio da interação de imunoglobulinas presentes no endotélio (ICAM-1 e VCAM-1) com integrinas, ocorrendo, então, a transmigração do mesmo, que é mediado pelo CD31. Os neutrófilos atravessam a membrana basal e migram seguindo um gradiente quimiotáxico que tem origem no local da lesão (KUMAR, ABBAS, FAUSTO, 2004).

As moléculas de adesão (CAM) são glicoproteínas expressas na superfície celular (GOMES, NETO, BISPO, 2009) que quando estimuladas apresentam uma superfície adesiva que permite a ligação de leucócitos recrutados durante a resposta inflamatória (KATO et al., 2005). Podem também ser responsabilizadas pela agressão direta ao endotélio, com edema, hemorragia e trombose dos microcapilares (FRENETTE; WAGNER, 1996).

Um dos tipos de CAM presentes no organismo, denominadas de moléculas de adesão intracelular-1 (ICAM-1), são moléculas de adesão compostas de polipeptídio transmembrana achadas no epitélio, nos fibroblastos e em células endoteliais e podem ser consideradas importantes armas no controle terapêutico de doenças que envolvem lesão endotelial (GOMES, NETO, BISPO, 2009). A ICAM-1 é normal- mente expressa em níveis baixos na superfície luminal das células endoteliais. A sua expressão é induzida por múltiplos fatores de estímulos biológicos, particularmente por citocinas inflamatórias (KATO et al., 2005) e é utilizada como um indicador de inflamação endotelial (OH et al., 2010).

Uma forma solúvel da ICAM-1 (sICAM-1) é liberada da ICAM-1 expressa nas células e secretada na circulação. Aumento de sICAM-1 pode ser uma consequência indireta de danos do tecido ou da inflamação (TEPPO et al., 2001). Em indivíduos sem manifestações clínicas de patologia cardiovascular, a sICAM-1 parece ser o melhor marcador de lesão endotelial (LIU et al., 2009).

O objetivo deste trabalho é correlacionar os níveis de ICAM-1 solúvel, em crianças com processo inflamatório com o desvio nuclear a esquerda, constituído por indivíduos de sexo feminino e masculino, com idade entre 0 e 18 anos e verificar o estado de ativação do endotélio em tais pacientes, de modo a investigar um possível aumento de sICAM-1 com o aumento de bastonetes.

\section{Materiais e Método}

$\mathrm{O}$ estudo foi realizado em pacientes com processo inflamatório agudo, sem doença crônica de 0 a 18 anos, de sexo feminino e masculino.

Houve análise de 37 amostras dos soros de pacientes, que foram obtidas por meio de amostras sanguíneas recolhidas para realização de exames de rotina e foram disponibilizadas pelo laboratório NeoLab de Toledo-PR. Amostras visualmente hemolisadas ou lipêmicas foram descartadas.

O hemograma dos pacientes foi realizado pelo laboratório e os resultados fornecidos a este estudo.

A determinação das concentrações séricas de sICAM-1 foi realizada no laboratório de Hematologia da Pontifícia Universidade Católica do Paraná (PUCPR) campus Toledo, por meio da técnica de enzimaimunoensaio (ELI$\mathrm{SA}$ ), seguindo os critérios exigidos na bula do kit da Invitrogen ${ }^{\circledR}$ e utilizando leitor de ELISA da marca Biotek ${ }^{\circledR}$ (modelo ELX800) para leitura das absorbâncias.

As análises estatísticas foram realizadas por meio da Análise da Variância (ANOVA) com critérios de significância $\mathrm{P}<0,05$ e coeficiente de correlação de Pearson.

Os prontuários foram disponibilizados após assinatura do 'Termo de compromisso de utilização de dados' (Apêndice A). O projeto foi aprovado pelo Comitê de Ética em Pesquisa em Seres Humanos do Hospital de Clínicas da UFPR, de acordo com o registro CEP: 2136.031/2010-02 (Anexo A).

\section{Resultados}

Foram analisados 37 pacientes que possuíam aumento de bastonetes e de acordo com as análises estatísticas pode-se notar que não há correlação entre o aumento de bastonete e o aumento da molécula sICAM-1 $(r=0,28)$ (Figura $1)$.

Dividindo-se os pacientes em dois grupos, um que possui sICAM-1 alterado e outro que possui sICAM-1 normal, observou-se por meio dos dados que estes possuem diferença notável de bastonetes $(\mathrm{p}=0,045)$, onde a média de bastonetes é maior no grupo sICAM-1 alterado (Figura 2). 
Também verificou-se uma correlação negativa fraca sobre o número de bastonetes e sICAM-1 em pacientes com esta molécula considerada normal $(\mathrm{r}=-0,49)$, ou seja, quanto mais se aumenta o número de bastonetes, diminui a concentração de sICAM-1 (Figura 3).

Figura 1: Ausência de correlação das concentrações de sICAM-1 versus a contagem de bastonetes em todos os grupos analisados.

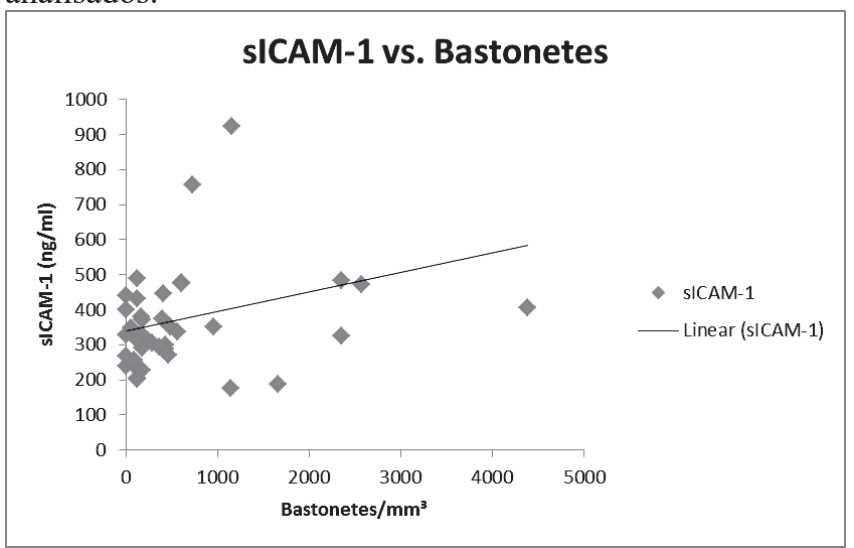

Fonte: a autora, 2014.

Figura 2: Comparação de número de bastonetes entre os dois grupos.

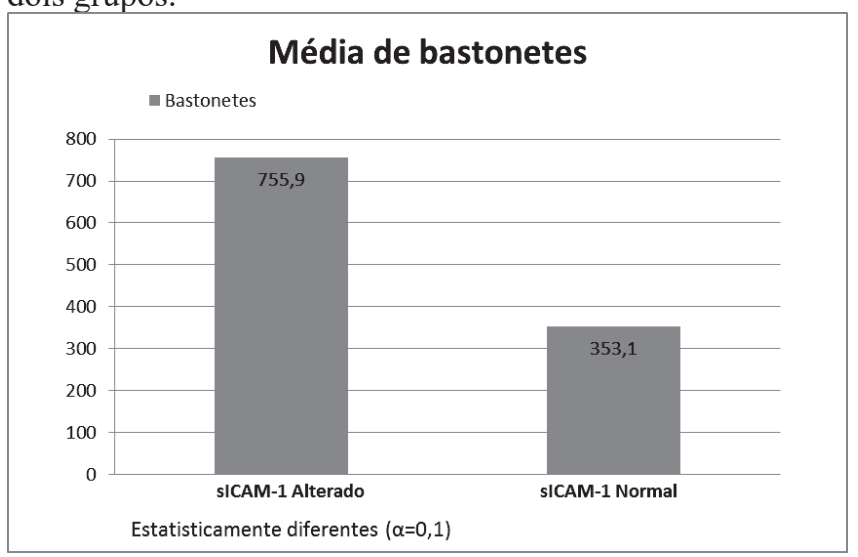

Fonte: a autora, 2014.

Figura 3: Correlação negativa das concentrações de sICAM-1 versus a contagem de bastonetes no grupo com sICAM-1 considerado normal.

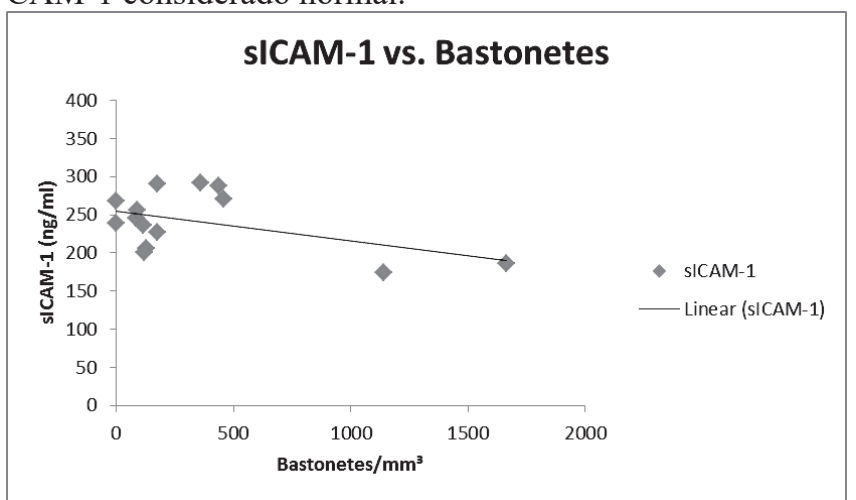

Fonte: a autora, 2014.

\section{Discussão}

As concentrações séricas de sICAM-1 nos pacientes analisados mostraram que não houve uma correlação entre o aumento do número de bastonetes e a dosagem de sICAM-1 (Figura 1), sugerindo que nestes casos não há relação entre as mesmas no processo inflamatório. Ao contrário de Shanley, Warner e Ward (1995) que apresentam as citocinas e o endotélio, bem como leucócitos e as moléculas de adesão como coordenadores de uma cascata de interações entre leucócitos e células endoteliais, o que resulta em lesão de tecidos.

Do mesmo modo que o estudo realizado por Teppo et al. (2001), mostra que houve uma elevação na expressão de sICAM-1 urinária em pacientes que apresentaram rejeição de transplante renal, e aponta que este aumento está ligado com a lesão tecidual ou simplesmente uma consequência da inflamação.

Segundo Cornbleet (2002), os neutrófilos são os leucócitos predominantes no sangue periférico e quando estimulados no processo inflamatório agudo por fatores de crescimento e citocinas, produzidas pelos macrófagos teciduais e outras células, induzem o aumento da produção de neutrófilos pela medula óssea. Essa produção demasiada faz com que a medula lance células imaturas no sangue periférico, aumentando assim a contagem de bastonetes. $\mathrm{O}$ aumento de bastonetes em todos os pacientes do nosso estudo e a elevação dos valores de sICAM-1 em alguns pacientes provavelmente esteja correlacionado a cascata inflamatória, porém como também observamos pacientes que não possuíram alteração de sICAM-1 (Figura 2), pode-se dizer que a mudança de expressão desta molécula sofra influência de outros fatores.

Tem sido relatado que as alterações de um único aminoácido no domínio de ICAM-1 podem influenciar fortemente a ligação dos seus ligandos in vivo e, consequentemente, os polimorfismos nesta região podem também fornecer um ganho e uma perda de função (DIAMOND et al., 1991). No estudo de Singh (2014) observou-se um maior nível de circulação de ICAM-1 em pacientes com neurocisticercose sintomáticos do que o de indivíduos assintomáticos e controles saudáveis. A diferença entre casos assintomáticos e controle foi pouca e notou-se que houve um aumento de ICAM-1 mesmo em pacientes considerados saudáveis. Isso mostra que a ICAM-1 pode estar presente em quantidades mensuráveis em todos os indivíduos, e em casos de resposta inflamatória sua concentração aumenta.

Uma possível explicação para não ter ocorrido o aumento de sICAM-1 nos pacientes do presente estudo, pode ser o uso de anti-inflamatórios esteroidais. Conforme Jilma et al. (2000), que fez um estudo para avaliar a concentração de ICAM-1 circulante após administração de duas doses de Dexametasona, foi observado que ambas as doses de Dexametasona produziram uma redução comparável em ICAM-1 circulante, enquanto houve aumento no número de neutrófilos em média de $460 \%$ e 176 \% em 48 horas após as altas e baixas doses de Dexametasona, respectivamente. Já um estudo realizado por Blann et al. (1998), sobre os efeitos agudos do tabagismo, revelou que em fumantes ocorre um aumento na contagem de leucócitos e ativação de neutrófilos, mas nenhuma mudança foi observada em relação ao sICAM-1. Para mensurar tais parâmetros, seria necessária a realização de 
uma anamnese na hora da coleta sanguínea.

\section{Conclusão}

Portanto, por meio dos estudos analisados foi possível notar que a presença de sICAM-1 aumentada normalmente esta ligada há casos inflamatórios, mas a mudança na sua expressão pode sofrer a influência de outros fatores. A ICAM-1 é observada em pequenas quantidades mensuráveis nos indivíduos e ocorrências inflamatórias elevam sua concentração.

O fato de não verificar o aumento na concentração de sICAM-1 não exclui um processo inflamatório e exige que outro rumo seja tomado para elucidar o quadro, tal como um questionamento sobre hábitos de vida do paciente e uso de medicamentos, para saber se a mesma não está sendo suprimida.

Uma possível explicação para não ter ocorrido o aumento de sICAM-1 nos pacientes do presente estudo, é de que estes estejam utilizando medicamentos do tipo anti-inflamatórios esteroidais.

Segundo a literatura, a sICAM-1 parece ser um bom marcador para lesão endotelial em pacientes com doenças cardiovasculares, sugere-se então um estudo mais aprofundado para estes casos.

\section{Conflito de Interesse}

Não há conflito de interesses.

\section{Suporte Financeiro}

O projeto foi financiado pelos seus autores.

\section{Agradecimentos}

Agradeço ao Paulo Roberto Merisio pela paciência e incentivo, e também a Vanessa Galeazzi pelo auxílio no desenvolvimento deste trabalho.

A PUC-PR pelas oportunidades, e aos professores desta universidade pelo apoio teórico.

\section{Referências}

ABBAS, A. K.; LICTMAN, A. H.; POBER, J. S. Imunologia celular e molecular. 4. ed. Rio de Janeiro: Revinter, 2002.

BLANN, A. D. The influence of acute smoking on leucocytes, platelets and the endothelium. Atherosclerosis, v. 141, n. 1, p. 133-139, 1998. Disponível em: <http://www. sciencedirect.com/science/article/pii/S0021915098001634>. Acesso em: 10 set. 2014.

BOTHA, A. J.; MOORE, F. A. et al. Postinjury neutrophil priming and activation states: therapeutic challenges.

Shock, v. 3, n. 3, mar. p.157-166, 1995.

CORNBLEET, J. Clinical utility of the band count. Clinics in laboratory medicine, v. 22, n. 1, p. 101-136, 2002.
DIAMOND, M. S. et al. Binding of the integrin Mac$1(\mathrm{CD} 11 \mathrm{~b} / \mathrm{CD} 18)$ to the third immunoglobulin-like domain of ICAM-1 (CD54) and its regulation by glycosylation. Cell, v. 65, n. 6, p. 961-971, 1991.

FERREIRA, M. C.; GROSSEMAN, S.; VIEIRA, R. S. O que os profissionais de saúde que lidam com saúde maternoinfantil deveriam saber sobre vacinas. Revista on-line da Associação Catarinense de Medicina, v. 2, n. 32, p. 70101, 2003. Disponível em: <http://www.acm.org.br/revista/ pdf/artigos/66.pdf $>$. Acesso em: 10 mar. 2014.

FILIZZOLA, V. C. C. et al. Fatores de risco para amigdalite aguda de repetição em crianças. Revista Brasileira Alergia e Imunopatologia, v. 21, n. 4, p. 100-104, 1998. Disponível em: <http://www.asbai.org.br/revistas/Vol214/risco.htm>. Acesso em: 11 mar. 2014.

FIOTTI, N. et al. Atherosclerosis and inflammation: patterns of cytokine regulation in patients with peripheral arterial disease. Atherosclerosis, v. 145, p. 51-60, 1999.

FRENETTE, P. S.; WAGNER, D. D. Adhesion molecules - blood vessels and blood cells. The new England journal of medicine, v. 335 p. 43-45, 1996. doi: 10.1056/ NEJM199607043350108.

GOMES, M. A. M.; MACÊDO NETO, N. C.; BISPO, I. G. A. Interleucina-6, moléculas de adesão intercelular-1 e Microalbuminúria na avaliação da lesão endotelial. Rev SOCERJ, v. 22, n. 6, p. 398-403, 2009.

HILL, A. et al. The effect of granulocyte-macrophage colony-stimulating fator on myeloid cells and its clinical applications. Journal of Leukocyte Biology, v. 58, p. 634-623, 1995. Disponível em: <http://www.jleukbio.org/ content/58/6/634.full.pdf>. Acesso em: 26 mar. 2014.

JILMA, B. et al. Dexamethasone lowers circulating E-selectin and ICAM-1 in healthy men. Journal of Laboratory and Clinical Medicine, v. 135, n. 3, p. 270274, 2000. Disponível em: <http:/www.sciencedirect.com/ science/article/pii/S0022214300044589>. Acesso em: 10 set. 2014.

JUNQUEIRA, L. C.; CARNEIRO, J. Histologia básica. 11. ed. Rio de Janeiro: Guanabara Koogan, 2008.

KATO, G. J. et al. Levels of soluble endothelium-derived adhesion molecules in patients with sickle cell disease are associated with pulmonary hypertension, organ dysfunction, and mortality. British Journal of Haematology, v. 130, n. 6, p. 943-53, 2005. Disponível em: <http://www.ncbi.nlm. nih.gov/pmc/articles/PMC2065864/>. Acesso em: 9 mar. 2014.

KUIJPERS, T.; ROOS, D. Neutrophils. Encyclopedia of life sciences, 2001. doi: 10.1038/npg.els.0001219.

KUMAR,V.; ABBAS, A. K; FAUSTO, N. Robbins \& Cotran: patologia - bases patológicas das doenças. 7. ed. 
Rio de janeiro: Elsevier, 2004.

LIU, M.; WANG, F.; WANG Y.; JIN, R. Atorvastatin improves endothelial function and cardiac performance in patients with dilated cardiomyopathy: the role of inflammation. Cardiovascular Drugs and Therapy, v. 23, n. 5, p. 369-376, 2009. doi: 10.1007/s10557-009-6186-3.

MORTENSEN, R. C-reactive protein, inflammation, and innate immunity. Immunologic Research, v. 24, p. 163176, 2001. Disponível em: <http://link.springer.com/article/ 10.1385\%2FIR \%3A24\%3A2\%3A163>. Acesso em: 26 abr. 2014.

$\mathrm{OH}, \mathrm{J}$. H. et al. A novel cyclin-dependent kinase inhibitor down-regulates tumor necrosis factor- $\alpha$ (TNF- $\alpha)$-induced expression of cell adhesion molecules by inhibition of NF- $\kappa \mathrm{B}$ activation in human pulmonary epithelial cells. Int. Immunopharmacol. v. 10, n. 5, p. 572-579, 2010.

OLIVEIRA, R. A. G. Hemograma: como fazer e interpretar. São Paulo: Livraria Paulista, 2007.

PARAHYBA, M. J. P. C.; SANTANA, L. R.; ALENCAR, M. J. M. Alguns aspectos avaliativos do hemograma em crianças com pneumonia. Revista RECCS, n.12, p.16-22, 2000. Disponível em: <http://ojs.unifor.br/index.php/RBPS/ article/viewFile/1987/1987>. Acesso em: 28 abr. 2014.

RIZZON, D. O Sistema imune do recém-nascido: destacando aspectos fetais e maternos. Revista de Pediatria SOPERJ, v. 12, n. 1, p. 12-15, 2011.

SINGH, A. Association of ICAM-1 K469E polymorphism with neurocysticercosis. Journal of Neuroimmunology, 2014. doi: 10.1016 / j.jneuroim.2014.07.018.

SHANLEY, T. P.; WARNER, R. L.; WARD, P. A. The role of cytokines and adhesion molecules in the development of inflammatory injury. Molecular Medicine Today, v. 1, n. 1, p. 40-45, 1995. doi: 10.1016/1357-4310(95)80019-0.

TEPPO, A. M. et al. Soluble intercellular adhesion molecule-1 (sICAM-1) After kidney transplantation: the origin and role of Urinary sICAM-1. Transplantation, $\mathrm{v}$. 71, n. 8, p. 1113-1119, 2001 